\title{
Cediranib: Überleben bei mCRC nicht verlängert
}

Trotz Oxaliplatin-basierter Therapien liegt das mediane Überleben bei metastasiertem kolorektalen Karzinom (mCRC) unter zwei Jahren. Zur Verbesserung der Wirksamkeit könnte eine potente Hemmung der Rezeptoren des vaskulären endothelialen Wachstumsfaktors (VEGF) beitragen.

$\mathrm{O}$ b Cediranib, ein hoch potenter Inhibitor aller drei VEGF-Rezeptoren, zusätzlich zur Chemotherapie das Überleben bei bisher unbehandeltem mCRC verbessert, wurde in der Phase-III-Studie Horizon II randomisiert und placebokontrolliert in zwei Dosierungen untersucht: Cediranib 20 oder $30 \mathrm{mg}$ pro Tag oder Placebo zusätzlich zur Chemotherapie mit Fluorouracil, Folinsäure und Oxaliplatin (FOLFOX) oder Capecitabin und Oxaliplatin (CAPOX). Nach der frühen Analyse dieser und zweier weiterer Studien mit Cediranib oder Bevacizumab zusätzlich zu FOLFOX6 bei bisher nicht behandeltem $\mathrm{mCRC}$ wurde die Studie nur mit der 20 mg-Dosis Cediranib fortgesetzt und die Patienten im Verhältnis 2:1 randomisiert entweder dem
Arm mit 20 mg Cediranib ( $\mathrm{n}=502)$ oder Placebo $(\mathrm{n}=358)$ zugeteilt. 26,2 \% erhielten dabei als Chemotherapie-Rückgrat FOLFOX4, 17,1\% FOLFOX6 und 56,7\% CAPOX. Primäre Endpunkte waren sowohl progressionsfreies (PFS) als auch Gesamtüberleben (OS).

Das PFS im Cediranib-Studienarm war mit 8,6 Monaten etwas länger als im Kontrollarm (8,3 Monate; Hazard Ratio [HR] 0,84; $\mathrm{p}=0,0121)$, aber beim medianen Gesamtüberleben gab es keinen Vorteil (19,7 vs. 18,9 Monate, HR 0,94; p $=0,5707$ ). Auch sekundäre Endpunkte wie objektive Ansprechrate, Dauer des Ansprechens oder Leber-Resektionsrate zeigten keinen Vorteil im CediranibArm. Die Nebenwirkungen im Interventions-Arm waren beherrschbar, die me-

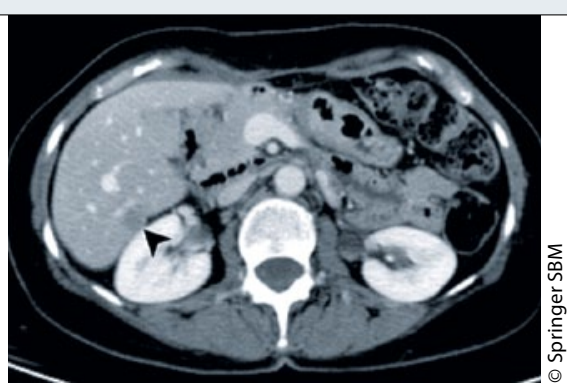

Viele Patienten mit mCRC entwickeln Lebermetastasen. Die Addition von Cediranib zur Chemotherapie führte in dieser Studie nicht zu mehr Resektionen von Lebermetastasen.

diane Dosisintensität der Chemotherapie aber um etwa $10 \%$ verringert.

Fazit: Die fehlende Überlegenheit bezüglich des Gesamtüberlebens und aller sekundären Endpunkte führt trotz eines geringen Vorteils beim PFS dazu, dass die Autoren Cediranib zusätzlich zur Chemotherapie in der Erstlinie beim mCRC nicht empfehlen. Friederike Klein

Hoff PM et al. Cediranib plus FOLFOX/CAPOX versus placebo plus FOLFOX/CAPOX in patients with previously untreated metastatic colorectal cancer: A randomized, double-blind, phase III study (HORIZON II). J Clin Oncol. 2012;30(29): 3596-603.

\section{Aflibercept - eine Alternative für die Antiangiogenese nach Oxaliplatin?}

Beim metastasierten kolorektalen Karzinom ( $m C R C$ ) erfolgt heute meist eine Fluoropyrimidin-basierte Chemotherapie, oft kombiniert mit Bevacizumab oder einem monoklonalen Antikörper gegen den vaskulären endothelialen Wachstumsfaktor (VEGF)-Rezeptor. Für Patienten mit Rezidiv ist möglicherweise wieder eine antiangiogenetische Therapie erfolgversprechend.

\footnotetext{
A flibercept ist ein Fusionsprotein mit den Bindungsanteilen von VEGFRezeptoren, das VEGF abfängt und so der Angiogenese im Tumorgewebe entgegenwirken soll. In einer Phase-III-Studie wurde die Effektivität und Sicherheit von Aflibercept zusätzlich zu FOLFIRI (Fluorouracil plus Folinsäure und Irinotecan) bei Patienten mit mCRC, die zuvor Oxaliplatin und in einigen Fällen auch Bevacizumab erhalten hatten, untersucht. Da zum Zeitpunkt der Studienplanung eine Therapie mit einem weiteren Wirkstoff zusätzlich zur Chemotherapie noch nicht etabliert war, erhielten die Patienten im
}

Kontrollarm zusätzlich zu FOLFIRI Placebo. Randomisiert erhielten die Patienten entweder Aflibercept (4 mg/kg KG i.v., $\mathrm{n}=612)$ oder Placebo $(\mathrm{n}=614)$ zusätzlich zu FOLFIRI alle zwei Wochen bis zum Progress oder dem Auftreten nicht tolerabler Toxizität.

Die Add-on-Therapie mit Aflibercept resultierte in einem signifikant besseren medianen Gesamtüberleben (primärer Endpunkt) im Verumarm (13,5 vs. 12,06 Monate, Hazard Ratio [HR] 0,817; p = 0,0032 ). Auch das progressionsfreie Überleben war signifikant besser unter der Addition von Aflibercept (6,90 vs.
4,67 Monate, HR 0,758; $p<0,0001$ ). Die Subgruppenanalye zeigte einen entsprechenden Trend in allen vor Studienbeginn definierten Subgruppen, auch in der mit Bevacizumab-Vorbehandlung. Auch die Ansprechrate war unter der Addition von Aflibercept signifikant höher als im Placebo-Arm (19,8 vs. $11,1 \%$; $\mathrm{p}=0,0001)$.

Aflibercept verstärkte aber die von FOLFIRI bekannten Toxizitäten und führte zudem zu den charakteristischen Nebenwirkungen, die von Antiangiogenese-Wirkstoffen bekannt sind (Hypertonie, Blutungen und arterielle wie venöse thromboembolische Ereignisse).

Fazit: Die Ergänzung der FOLFIRI-Therapie des Oxaliplatin-vorbehandelten mCRC um Aflibercept führt zu einem statistisch signifikanten Überlebensvorteil.

Friederike Klein

Van Cutsem E et al. Addition of Aflibercept to Fluorouracil, Leucovorin, and Irinotecan Improves Survival in a Phase III Randomized Trial in Patients With Metastatic Colorectal Cancer Previously Treated With an Oxaliplatin-Based Regimen. J Clin Oncol. 2012;30(28):3499-506. 